\title{
Optimal PWM Control of Switched-Capacitor DC/DC Power Converters via Model Transformation and Enhancing Control Techniques
}

\author{
Charlotte Yuk-Fan $\mathrm{Ho}^{1}$, Student Member, IEEE, Bingo Wing-Kuen Ling ${ }^{2}$, Yan-Qun Liu ${ }^{3}$, Peter \\ Kwong-Shun Tam ${ }^{4}$, and Kok-Lay Teo ${ }^{5}$, Senior Member, IEEE
}

\begin{abstract}
This paper presents an efficient and effective method for an optimal pulse width modulated (PWM) control of switched-capacitor DC/DC power converters. Optimal switching instants are determined based on minimizing the output ripple magnitude, the output leakage voltage and the sensitivity of the output load voltage with respect to both the input voltage and the load resistance. This optimal PWM control strategy has several advantages over conventional PWM control strategies: 1) It does not involve a linearization, so a large signal analysis is performed. 2) It guarantees the optimality. The problem is solved via both the model transformation and the optimal enhancing control techniques. A practical example of the PWM control of a switched-capacitor DC/DC power converter is presented.
\end{abstract}

Index Terms-Optimal PWM control, switched-capacitor DC/DC power converters, model transformation technique, optimal enhancing control technique.

\section{INTRODUCTION}

$\mathrm{M}$ ANY consumer and industrial electronic products, such as LCD drivers [1], pocket computer systems [2], etc, require more than one voltage sources. Hence, DC/DC power converters are required for these applications. For some applications, DC/DC power converters require both low EMI and EMC. In these cases, inductors are not recommended to be

Manuscript received February 1, 2006; revised June 28, 2007. This work was supported by a research grant (project number G-YD26) from The Hong Kong Polytechnic University, the Centre for Multimedia Signal Processing, The Hong Kong Polytechnic University, the CRGC grant (project number PolyU 5105\01E) from the Research Grants Council of Hong Kong, as well as a research grant from Australian Research Council.

Charlotte Yuk-Fan Ho is with the School of Mathematical Sciences, Queen Mary, University of London (e-mail: c.ho@qmul.ac.uk).

Bingo Wing-Kuen Ling is with the department of Electronic Engineering, King's College London (e-mail: wing-kuen.ling@kcl.ac.uk).

Yan-Qun Liu is with the Department of Mathematics and Statistics, Royal Melbourne Institute of Technology (e-mail: yanqun.liu@rmit.edu.au).

Peter Kwong-Shun Tam is with the Department of Electronic and Information Engineering, The Hong Kong Polytechnic University (e-mail: enptam@eie.polyu.edu.hk).

Kok-Lay Teo is with the Department of Mathematics and Statistics, Curtin University of Technology (e-mail: K.L.Teo@curtin.edu.au).

Copyright (c) 2007 IEEE. Personal use of this material is permitted. However, permission to use this material for any other purposes must be obtained from the IEEE by sending an email to pubs-permission@ieee.org. employed for the realization. Capacitors are used instead. These DC/DC power converters are called switched-capacitor DC/DC power converters or inductorless $\mathrm{DC} / \mathrm{DC}$ power converters. As they could achieve both low EMI and EMC requirements for many consumer and industrial electronic products, solving unaddressed problems on switched-capacitor DC/DC power converters would be beneficial to the community.

Switched-capacitor DC/DC power converters consist of some switches and capacitors, in which the capacitors are energy storage components. At certain switching instants, some of the capacitors are switched to an input energy source so that energy is stored in these capacitors. At the same time, another set of capacitors are switched to the output load and deliver energy. When the switches turn on and turn off alternatively, those capacitors which have released energy before, are now switched to the input energy source and charged up, whilst, those capacitors which have stored energy before, are now switched to the output load and deliver energy. Because of this operating principle, ripples usually occur at the output load voltage, while the ripple magnitude of the output load voltage is dependent on the capacitances of the capacitors, the resistance of the load, and the switching instants. Since almost all the applications require a steady voltage for their operations, the output ripple magnitude should be minimized.

Simultaneously, reverse-recovery currents of the diodes and the transistors, as well as their parasitic capacitances, would make the output load voltage jumping at the switching instants. Such jumps at the output load voltage are called the output leakage voltage. Similarly, the output leakage voltage should also be minimized.

At the same time, the load resistance would affect the time constant of the discharging circuit, and the input voltage would affect the voltages across the capacitors in the charging circuit, both the load resistance and the input voltage would affect the output load voltage. From the practical point of view, the output load voltage should remain unchanged even though the load resistance and the input voltage are changed. Hence, it is preferred to have a low sensitivity of the output load voltage with respect to both the input voltage and the load resistance. 
Therefore, the sensitivity of the output load voltage with respect to both the input voltage and the load resistance should also be minimized.

There are some existing methods for minimizing the output ripple magnitude of a switched-capacitor $\mathrm{DC} / \mathrm{DC}$ power converter, such as multirate control method [16], LQR control methods [17]-[19], intelligent control method [20], and PWM control methods [21]-[25], etc. On the other side, some methods have been proposed for regulating the output load voltage, such as PWM control method [32], noise shaping method [33], pseudo-continuous control method [34], interleaved discharging method [35], and constant frequency charge pump method [36], etc. Among them, PWM control methods are the common method for both minimizing the output ripple magnitude and regulating the output load voltage subject to the change in the input voltage and the load resistance. This is because these methods exploit both the switching and the nonlinear phenomena of the switched-capacitor DC/DC power converters [21]-[25], [32]. The implementations of the PWM control methods can also be simple [23]. However, there is one fundamental question for the PWM control methods: How to determine the switching instants so that the output ripple magnitude, the output leakage voltage and the sensitivity of the output load voltage with respect to both the input voltage and the load resistance are minimized? This problem is not trivial and has not been completely solved yet. The conventional PWM control methods [21]-[25] are based on small signal approximation. That is, the switched-capacitor DC/DC power converter is modeled as a linear time invariant system via perturbating the equations around a fixed point or employing a state space averaging model. Nevertheless, these approaches are only a local approximation of the problem and the obtained switching instants are not guaranteed to be optimal. For conventional optimal control methods [17]-[19], optimal control signals are determined so that a smooth cost function is minimized subject to some smooth constraints. Note that this optimal PWM control is different from the conventional optimal control. The goal for this optimal PWM control is to determine the optimal switching instants, but not to determine the optimal control input signals. Moreover, the switched-capacitor DC/DC power converter switches among different topologies, so the switched-capacitor DC/DC power converter is actually time-varying. Furthermore, initial conditions of the circuit corresponding to each topology are non-zero at the steady state, so the switched-capacitor $\mathrm{DC} / \mathrm{DC}$ power converter is nonlinear. An inadequate selection of the switching instants and the nonlinear time-varying nature of the switched-capacitor DC/DC power converters may result to instability problems [3]-[5] or the occurrence of chaotic behaviors [6]-[15].

The paper is organized as follows. The system model and the problem formulation are presented in, respectively, Section II and Section III. A solution method is discussed in Section IV. A practical example of PWM control of a switched-capacitor DC/DC power converter is presented in Section V. Finally, a conclusion is drawn in Section VI.

\section{SySTEM MODEL}

Consider a switched-capacitor DC/DC power converter in which it switches among different topologies with the dynamics of the circuit corresponding to each topology is characterized by an affine linear time invariant model. Without loss of generality, we assume that there are totally $N$ topologies and $N-1$ switching instants within a time period, denoted as $[0, T)$. Those switching instants are denoted as $t_{i}$ for $i=1,2, \cdots, N-1$. Denote $t_{0}=0$ and $t_{N}=T$. During the $i^{\text {th }}$ time interval $\left[t_{i-1}, t_{i}\right)$ for $i=1,2, \cdots, N$, let the state space representation of the circuit corresponding to the $i^{\text {th }}$ topology be $\mathrm{S}_{i}=\left(\mathbf{A}_{i}, \mathbf{B}_{i}, \mathbf{C}_{i}, \mathbf{D}_{i}\right)$ as follows:

$$
\begin{aligned}
\frac{d \mathbf{x}(t)}{d t} & =\mathbf{A}_{i} \mathbf{x}(t)+\mathbf{B}_{i} \mathbf{u}(t), \\
y(t) & =\mathbf{C}_{i} \mathbf{x}(t)+\mathbf{D}_{i} \mathbf{u}(t),
\end{aligned}
$$

where $\mathbf{A}_{i} \in \mathfrak{R}^{n \times n}, \mathbf{B}_{i} \in \mathfrak{R}^{n \times p}, \mathbf{C}_{i} \in \mathfrak{R}^{1 \times n}$ and $\mathbf{D}_{i} \in \mathfrak{R}^{1 \times p}$, in which $\mathfrak{R}^{k \times q}$ represents the set of $k \times q$ real matrices, the state vector $\mathbf{x}(t) \in \mathfrak{R}^{n \times 1}$ represents the voltages across the capacitors, $y(t) \in \mathfrak{R}$ and $\mathbf{u}(t) \in \mathfrak{R}^{p \times 1}$ represent the voltage across the load resistor and the input voltages of the switched-capacitor DC/DC power converter, respectively, where $\mathfrak{R}^{n}$ and $\mathfrak{R}$ denote the sets of $n$-dimensional real vectors and real numbers, respectively.

\section{Problem Formulation}

The output ripple magnitude of the switched-capacitor DC/DC power converter at the $i^{\text {th }}$ time interval $\left[t_{i-1}, t_{i}\right)$ for $i=1,2, \cdots, N$ is $\max _{t \in\left[t_{i-1}, t_{i}\right)} y(t)-\min _{t \in\left[t_{i-1}, t_{i}\right)} y(t)$. Hence, the output ripple magnitude of the switched-capacitor DC/DC power converter can be defined as $\max _{1 \leq i \leq N}\left(\max _{t \in\left[t_{i-1}, t_{i}\right)} y(t)-\min _{t \in\left[t_{i-1}, t_{i}\right)} y(t)\right)$. For a switched-capacitor DC/DC power converter, it is preferred to have small values of the output ripple magnitude. Hence, $\max _{1 \leq i \leq N}\left(\max _{t \in\left[t_{i-1}, t_{i}\right)} y(t)-\min _{t \in\left[t_{i-1}, t_{i}\right)} y(t)\right)$ should be minimized.

At the $i^{\text {th }}$ switching instant, where $i=1,2, \cdots, N-1$, a state leakage voltage, denoted as $\Delta \mathbf{x}\left(t_{i}-0\right)$, is expected. Without loss of generality, it can be expressed as:

$$
\Delta \mathbf{x}\left(t_{i}-0\right)=\mathbf{x}\left(t_{i}\right)-\mathbf{x}\left(t_{i}-0\right)=\boldsymbol{\varphi}_{i}\left(\mathbf{x}\left(t_{i}-0\right)\right)-\mathbf{x}\left(t_{i}-0\right),
$$

where $\boldsymbol{\varphi}_{i}$ for $i=1,2, \cdots, N-1$ is supposed to be a continuously differentiable function. In general, the state leakage voltage is too complicated to be represented mathematically. Instead, we approximate $\boldsymbol{\varphi}_{i}$ for $i=1,2, \cdots, N-1$ experimentally. Firstly, capacitors are charged up to different voltages. Then these capacitors are detached from the switched-capacitor DC/DC power converter and the voltage changes across these capacitors are recorded instantaneously. The voltage changes and the voltages across these capacitors correspond to the values of $\Delta \mathbf{x}\left(t_{i}-0\right)$ and $\mathbf{x}\left(t_{i}-0\right)$, respectively. By fitting a 
polynomial to approximate the relationship between $\Delta \mathbf{x}\left(t_{i}-0\right)$ and $\mathbf{x}\left(t_{i}-0\right)$, an approximation of $\boldsymbol{\varphi}_{i}$ for $i=1,2, \cdots, N-1$ could be obtained accordingly. And the output load voltage at the switching instants is:

$$
y\left(t_{i}\right)=\mathbf{C}_{i} \boldsymbol{\varphi}_{i}\left(\mathbf{x}\left(t_{i}-0\right)\right)+\mathbf{D}_{i} \mathbf{u}\left(t_{i}\right)=y\left(t_{i}-0\right)+\Delta y\left(t_{i}-0\right)
$$

for $i=1,2, \cdots, N-1$. The output leakage voltage at the $i^{\text {th }}$ switching instant for $i=1,2, \cdots, N-1$ is $\left|y\left(t_{i}\right)-y\left(t_{i}-0\right)\right|$, so the output leakage voltage of the switched-capacitor DC/DC power converter can be defined as $\max _{1 \leq i \leq N-1}\left|y\left(t_{i}\right)-y\left(t_{i}-0\right)\right|$, where $|\cdot|$ denotes the absolute operator. Similarly, it is preferred to have small values of the output leakage voltage. Hence, $\max _{1 \leq i \leq N-1}\left|y\left(t_{i}\right)-y\left(t_{i}-0\right)\right|$ should be minimized.

Denote the load resistance as $R_{L}$ and the gradient operator as $\nabla$. Then, the sensitivity of the output load voltage with respect to the load resistance and the input voltage at a particular time instant $t$ within the $i^{\text {th }}$ time interval $\left[t_{i-1}, t_{i}\right)$ for $i=1,2, \cdots, N$ are, respectively, $\frac{d y(t)}{d R_{L}}$ and $\left|\nabla_{\mathbf{u}(t)} y(t)\right|_{\infty}$. Hence, the sensitivity of the output load voltage with respect to the load resistance and the input voltage can be defined as, respectively, $\max _{1 \leq i \leq N} \max _{t \in t_{i-1}, t_{i}}\left|\frac{d y(t)}{d R_{L}}\right|$ and $\max _{1 \leq i \leq N} \max _{t \in\left[t_{i-1}, t_{i}\right)}\left|\nabla_{\mathbf{u}(t)} y(t)\right|_{\infty}$. As discussed in

Section I, these two terms should be minimized.

$$
\begin{aligned}
\text { Let } \xi=\left[t_{1}, t_{2}, \cdots, t_{N-1}\right]^{T} \in \mathfrak{R}^{N-1}, \text { and } \\
\\
\qquad \mathfrak{I}=\left\{\xi \in \mathfrak{R}^{N-1}: t_{0} \leq t_{1} \leq t_{2} \leq \cdots \leq t_{N-1} \leq t_{N}\right\} .
\end{aligned}
$$

The optimal PWM control problem becomes the determination of the optimal switching instants $\xi \in \mathfrak{I}$ so that the output ripple magnitude, the output leakage voltage and the sensitivity of the output load voltage with respect to both the load resistance and the input voltage are minimized. This problem is a multiple-objectives optimization problem. The most common method for solving a multiple-objectives optimization problem is to convert it to a single-objective optimization problem with the cost function being a weighted combination of these multiple objectives. Denote $\beta_{i}^{\prime}$ for $i=1,2, \cdots, 4$ as the weights for combining these multiple objectives. Then the problem can be expressed as follows:

\section{Problem (P)}

$$
\begin{aligned}
& \min _{\xi \in \mathfrak{I}} J(\xi)=\beta_{1}^{\prime} \max _{1 \leq i \leq N}\left(\max _{t \in\left[t_{i-1}, t_{i}\right.} y(t)-\min _{t \in\left[t_{i-1}, t_{i}\right)} y(t)\right)+\beta_{2}^{\prime} \max _{1 \leq i \leq N-1}\left|y\left(t_{i}\right)-y\left(t_{i}-0\right)\right|, \\
& +\beta_{3}^{\prime} \max _{1 \leq i \leq N} \max _{\left.t \in t_{i-1}, t_{i}\right)}\left|\frac{d y(t)}{d R_{L}}\right|+\beta_{4}^{\prime} \max _{1 \leq i \leq N} \max _{\left.t \in t_{i-1}, t_{i}\right)}\left|\nabla_{\mathbf{u}(t)} y(t)\right|_{\infty} \\
& \text { subject to } \frac{d \mathbf{x}(t)}{d t}=\mathbf{A}_{i} \mathbf{x}(t)+\mathbf{B}_{i} \mathbf{u}(t) \text { for } t \in\left[t_{i-1}, t_{i}\right) \text { and for } i=1,2, \cdots, N, \\
& \quad y(t)=\mathbf{C}_{i} \mathbf{x}(t)+\mathbf{D}_{i} \mathbf{u}(t) \text { for } t \in\left[t_{i-1}, t_{i}\right) \text { and for } i=1,2, \cdots, N, \\
& \quad \mathbf{x}\left(t_{0}\right)=\mathbf{x}_{0},
\end{aligned}
$$

and

$$
\mathbf{x}\left(t_{i}\right)=\boldsymbol{\varphi}_{i}\left(\mathbf{x}\left(t_{i}-0\right)\right)=\mathbf{x}\left(t_{i}-0\right)+\Delta \mathbf{x}\left(t_{i}-0\right) \text { for } i=1,2, \cdots, N-1 .(4
$$

\section{Solution Method}

\section{A. Model transformation method}

Problem (P) is a min-max problem, which involves a non-differentiable cost function. To address this problem, since 1) minimizing the maximum value of a function $f(t, \mathbf{u})$ over the index set $\Omega$ is equivalent to minimizing a parameter $v$ subject to all functional values over the index set being smaller than or equal to $v$, that is $\min _{\mathbf{u}} \max _{t \in \Omega} f(t, \mathbf{u})$ is equivalent to $\min _{\mathbf{u}}$ $v$ subject to $f(t, \mathbf{u}) \leq v \quad \forall t \in \Omega$, and 2) maximizing the minimum value of a function over the index set is equivalent to minimizing a parameter $v$ subject to the negative of all functional values over the index set being smaller than or equal to $v$, that is $\max _{\mathbf{u}} \min _{t \in \Omega} f(t, \mathbf{u})$ is equivalent to $\min _{\mathbf{u}} v$ subject to - $f(t, \mathbf{u}) \leq v \quad \forall t \in \Omega$, minimizing the maximum absolute value of a function over the index set is equivalent to minimizing a parameter $v$ subject to both the positive and the negative of all functional values over the index set being smaller than or equal to $v$, that is $\min _{\mathbf{u}} \max _{t \in \Omega}|f(t, \mathbf{u})|$ is equivalent to $\min _{\mathbf{u}} v$ subject to both $f(t, \mathbf{u}) \leq v \quad \forall t \in \Omega$ and $-f(t, \mathbf{u}) \leq v \quad \forall t \in \Omega$. Also, minimizing the difference between the maximum and minimum values of a function over the index set is equivalent to minimizing a parameter $v_{1}$ subject to all functional values over the index set being smaller than or equal to $v_{1}$ and minimizing a parameter $v_{2}$ subject to the negative of all functional values over the index set being smaller than or equal to $v_{2}$, that is $\min _{\mathbf{u}}\left(\max _{t \in \Omega} f(t, \mathbf{u})-\min _{t \in \Omega} f(t, \mathbf{u})\right)$ is equivalent to $\min _{\mathbf{u}} v_{1}$ subject to $f(t, \mathbf{u}) \leq v_{1} \forall t \in \Omega$ and $\min _{\mathbf{u}} v_{2}$ subject to $-f(t, \mathbf{u}) \leq v_{2} \quad \forall t \in \Omega$.

Hence, Problem (P) can be converted into a smooth continuous constrained optimization problem. Denote the set of the fifth dimensional real vectors as $\mathfrak{R}^{5}$. Let $\mathbf{v} \equiv\left[\begin{array}{lllll}v_{1} & v_{2} & v_{3} & v_{4} & v_{5}\end{array}\right]^{T}$ be a vector in $\mathfrak{R}^{5}$. Similarly, denote $\beta_{i}$ for $i=1,2, \cdots, 5$ as the weights for combining the multiple objectives. Denote $\mathbf{u}(t) \equiv\left[\begin{array}{lll}u_{1}(t) & \cdots & u_{p}(t)\end{array}\right]^{T}$. Then Problem (P) is equivalent to the following problem:

\section{Problem (Q)}

$\min _{\xi \in \Im, \mathbf{v} \in \Re^{5}} \hat{J}(\xi, \mathbf{v})=\sum_{i=1}^{5} \beta_{i} v_{i}$,

subject to $y(t) \leq v_{1} \forall t \in\left[t_{i-1}, t_{i}\right)$ and for $i=1,2, \cdots, N$,

$-y(t) \leq v_{2} \quad \forall t \in\left[t_{i-1}, t_{i}\right)$ and for $i=1,2, \cdots, N$,

$y\left(t_{i}\right)-y\left(t_{i}-0\right) \leq v_{3}$ for $i=1,2, \cdots, N-1$,

$y\left(t_{i}-0\right)-y\left(t_{i}\right) \leq v_{3}$ for $i=1,2, \cdots, N-1$,

$\frac{d y(t)}{d R_{L}} \leq v_{4} \quad \forall t \in\left[t_{i-1}, t_{i}\right)$ and for $i=1,2, \cdots, N$,

$-\frac{d y(t)}{d R_{L}} \leq v_{4} \forall t \in\left[t_{i-1}, t_{i}\right)$ and for $i=1,2, \cdots, N$,

$\frac{\partial y(t)}{\partial u_{k}(t)} \leq v_{5} \forall t \in\left[t_{i-1}, t_{i}\right)$, for $i=1,2, \cdots, N$ and for $k=1,2, \cdots, p$ 
$-\frac{\partial y(t)}{\partial u_{k}(t)} \leq v_{5} \quad \forall t \in\left[t_{i-1}, t_{i}\right)$, for $i=1,2, \cdots, N$ and for $k=1,2, \cdots, p$,

$\frac{d \mathbf{x}(t)}{d t}=\mathbf{A}_{i} \mathbf{x}(t)+\mathbf{B}_{i} \mathbf{u}(t)$ for $t \in\left[t_{i-1}, t_{i}\right)$ and for $i=1,2, \cdots, N$,

$y(t)=\mathbf{C}_{i} \mathbf{x}(t)+\mathbf{D}_{i} \mathbf{u}(t)$ for $t \in\left[t_{i-1}, t_{i}\right)$ and for $i=1,2, \cdots, N$,

$\mathbf{x}(0)=\mathbf{x}_{0}$,

and

$\mathbf{x}\left(t_{i}\right)=\boldsymbol{\varphi}_{i}\left(\mathbf{x}\left(t_{i}-0\right)\right)=\mathbf{x}\left(t_{i}-0\right)+\Delta \mathbf{x}\left(t_{i}-0\right)$ for $i=1,2, \cdots, N-1$. (

This approach for solving optimization problems with a min-max cost function is called the model transformation method [26].

\section{B. Enhancing control method}

Since the switching instants are generally located nonuniformly in the switching period, it is required to map the original time instant $t$ to a transformed time instant $s$ so that there is a one-to-one correspondence between $t$ and $s$, and the transformed switching instants are uniformly located in the real number line. To achieve this goal, we need the original switching instants $t_{i}$ for $i=1,2, \cdots, N-1$ being mapped to the consecutive integer instants $i$, and $\forall t \in\left[t_{i-1}, t_{i}\right), t$ being mapped linearly to $s \in[i-1, i)$. First, denote $\chi_{[i-1, i)}(s)=\left\{\begin{array}{ll}1 & s \in[i-1, i) \\ 0 & \text { otherwise }\end{array}\right.$ for $i=1,2, \cdots, N$ as a unit square pulse with the time support located at the time interval $[i-1, i)$. Define the switching durations as $\tau_{i} \equiv t_{i}-t_{i-1}$ for $i=1,2, \cdots, N$. Define a vector $\boldsymbol{\tau}=\left[\begin{array}{llll}\tau_{1}, & \tau_{2}, & \cdots, & \tau_{N}\end{array}\right]^{T} . \quad$ Define $\mu(\cdot \mid \tau):[0, N) \rightarrow \mathfrak{R}$ as a rectangular pulse train with the magnitude of the pulse train within the time interval $[i-1, i)$ being constant and equal to $\tau_{i}$ for $i=1,2, \cdots, N$, that is:

$$
\mu(s \mid \tau)=\sum_{i=1}^{N} \tau_{i} \chi_{[i-1, i)}(s) \text {. }
$$

Let $\Lambda=\left\{\mu(\cdot \mid \tau): \tau_{i} \geq 0\right.$ for $i=1, \cdots, N$ and $\left.\sum_{i=1}^{N} \tau_{i}=T\right\}$. Define $U: \tau \mapsto \mu(\cdot \mid \tau)$ as follows:

$$
U(\tau)=\mu \text {. }
$$

$U$ is a function that maps the vector $\tau$ to the rectangular pulse train function $\mu(s \mid \tau)$. It is worth noting that the switching instants of $\mu(s \mid \tau)$ are uniformly located at the consecutive integer instants $s=1,2, \cdots, N-1$. The original switching durations $\tau_{i}$ for $i=1,2, \cdots, N$ are the magnitudes of the function $\mu(s \mid \tau)$ for $s \in[i-1, i)$, that is $\mu(s \mid \tau)=\tau_{i}$ for $s \in[i-1, i)$ and for $i=1,2, \cdots, N$. However, $\mu(s \mid \tau)$ is not continuous and the relationship between $t$ and $s$ is not invertible. To address this problem, define $t(\cdot \mid \mu):[0, N) \rightarrow[0, T)$ as a piecewise linear function with the slope of the function in the time interval $[i-1, i)$ being constant and equal to $\tau_{i}$ for $i=1,2, \cdots, N$, that is:

$$
t(s \mid \mu)=\int_{0}^{s} \mu(\theta \mid \tau) d \theta \text {. }
$$

5i) Noting that the switching instants of $t(s \mid \mu)$ are also uniformly located at the consecutive integer instants $s=1,2, \cdots, N-1$, the original switching instants $t_{i}$ for $i=1,2, \cdots, N-1$ are the value of $t(s \mid \mu)$ at the switching instants $s=1,2, \cdots, N-1$, that is $t(i \mid \mu)=t_{i}$ for $i=1,2, \cdots, N-1, t(s \mid \mu)$ is continuous and there is a linear relationship between any time instant $t \in\left[t_{i-1}, t_{i}\right)$ and ) a nonnegative real number $s \in[i-1, i)$ for $i=1,2, \cdots, N$. Let $\mathbf{z}(s \mid \mu)$ and $w(s \mid \mu)$ be, respectively, the transformed state vector and the transformed output, in which the switching instants of both $\mathbf{z}(s \mid \mu)$ and $w(s \mid \mu)$ are uniformly located at the consecutive integer instants, that is $\mathbf{z}(s \mid \mu)=\mathbf{x}(t(s \mid \mu) \mid \boldsymbol{\tau})$ and $w(s \mid \mu)=\mathbf{y}(t(s \mid \mu) \mid \boldsymbol{\tau})$. Since $\frac{d}{d s} t(s \mid \mu)=\mu(s \mid \boldsymbol{\tau})$ for $i-1<s<i$ and for $i=1,2, \cdots, N$, define the following problem:

\section{Problem (R)}

$\min _{\xi \in \Im, \mathbf{I} \in \Re^{5}} \widetilde{J}(\boldsymbol{\xi}, \mathbf{v})=\sum_{i=1}^{5} \beta_{i} v_{i}$,

subject to $w(t(s \mid \mu)) \leq v_{1}$ for $s \in[i-1, i)$ and for $i=1,2, \cdots, N,(9 b)$

$-w(t(s \mid \mu)) \leq v_{2}$ for $s \in[i-1, i)$ and for $i=1,2, \cdots, N$,

$w(t(i \mid \mu))-w(t(i-0 \mid \mu)) \leq v_{3}$ for $i=1,2, \cdots, N-1$,

$w(t(i-0 \mid \mu))-w(t(i \mid \mu)) \leq v_{3}$ for $i=1,2, \cdots, N-1$,

$\frac{d w(t(s \mid \mu))}{d R_{L}} \leq v_{4}$ for $s \in[i-1, i)$ and for $i=1,2, \cdots, N$,

$-\frac{d w(t(s \mid \mu))}{d R_{L}} \leq v_{4}$ for $s \in[i-1, i)$ and for $i=1,2, \cdots, N$,

$\frac{\partial w(t(s \mid \mu))}{\partial u_{k}(t(s \mid \mu))} \leq v_{5}$ for $s \in[i-1, i)$, for $i=1,2, \cdots, N$ and for

$k=1,2, \cdots, p$,

$-\frac{\partial w(t(s \mid \mu))}{\partial u_{k}(t(s \mid \mu))} \leq v_{5}$ for $s \in[i-1, i)$, for $i=1,2, \cdots, N$ and for

$k=1,2, \cdots, p$,

$\frac{d}{d s} \mathbf{z}(s \mid \mu)=\mu(s \mid \boldsymbol{\tau})\left(\mathbf{A}_{i} \mathbf{z}(t(s \mid \mu))+\mathbf{B}_{i} \mathbf{u}(t(s \mid \mu))\right) \quad$ for $\quad s \in[i-1, i)$

and for $i=1,2, \cdots, N$,

$w(t(s \mid \mu))=\mathbf{C}_{i} \mathbf{z}(t(s \mid \mu))+\mathbf{D}_{i} \mathbf{u}(t(s \mid \mu))$ for $s \in[i-1, i)$ and for

$i=1,2, \cdots, N$,

$$
\mathbf{z}(t(0 \mid \mu))=\mathbf{x}_{0}
$$

and

$\mathbf{z}(t(i \mid \mu))=\boldsymbol{\varphi}_{i}(\mathbf{z}(t(i-0 \mid \mu)))=\mathbf{z}(t(i-0 \mid \mu))+\Delta \mathbf{z}(t(i-0 \mid \mu))$ for $i=1,2, \cdots, N-1$.

\section{Theorem 1}

Problem $(\mathrm{Q})$ and Problem $(\mathrm{R})$ are equivalent in the sense that $\left(\xi^{*}, \mathbf{v}^{*}\right)$ is a solution of Problem (Q) if and only if $\left(\mu^{*}, \mathbf{v}^{*}\right)$ is a solution of Problem (R), where $U\left(\tau^{*}\right)=\mu^{*}$, in which $\boldsymbol{\tau}^{*}=\left[\tau_{1}^{*}, \quad \tau_{2}^{*}, \cdots, \tau_{N}^{*}\right]^{T}, \xi^{*}=\left[t_{1}^{*}, t_{2}^{*}, \cdots, t_{N-1}^{*}\right]^{T}$ and $\tau_{i}^{*}=t_{i}^{*}-t_{i-1}^{*}$. Furthermore, $\hat{J}\left(\xi^{*}, \mathbf{v}^{*}\right)=\widetilde{J}\left(\mu^{*}, \mathbf{v}^{*}\right)$.

Proof: 
It is clear that $\left(\xi^{*}, \mathbf{v}^{*}\right)$ satisfies $(5 b)-(5 m)$ if and only if $U\left(\tau^{*}\right)=\mu^{*}$. Hence, $\left(\xi^{*}, \mathbf{v}^{*}\right)$ is a solution of Problem (Q) if and only if $\left(\mu^{*}, \mathbf{v}^{*}\right)$ is a solution of Problem (R). Furthermore, from (5a) and (9a), we can easily verify that $\hat{J}\left(\xi^{*}, \mathbf{v}^{*}\right)=\widetilde{J}\left(\mu^{*}, \mathbf{v}^{*}\right)$.

This method is called the enhancing control method [26]-[28].

\section{Solving state jump problem}

The discontinuities of the state variables are handled by computing the state variables segment by segment [29]. The value of the initial condition of the current segment can be computed by the value of the state variables at the end of the previous segment. To find $\mathbf{z}(t(i \mid \mu))$ for $i=1,2, \cdots, N-1$, we first compute the value of $\mathbf{z}(t(1-0 \mid \mu))$ using the initial condition $\mathbf{z}(t(0 \mid \mu))$. Then $\mathbf{z}(t(1 \mid \mu))$ can be evaluated by the equation $\mathbf{z}(t(1 \mid \mu))=\boldsymbol{\varphi}_{1}(\mathbf{z}(t(1-0 \mid \mu)))$. Similarly, $\mathbf{z}(t(2-0 \mid \mu))$ can be computed using $\mathbf{z}(t(1 \mid \mu))$, and $\mathbf{z}(t(2 \mid \mu))$ can be evaluated by the equation $\mathbf{z}(t(2 \mid \mu))=\boldsymbol{\varphi}_{2}(\mathbf{z}(t(2-0 \mid \mu)))$. These procedures are repeated until all the values of $\mathbf{z}(t(i \mid \mu))$ for $i=1,2, \cdots, N-1$ are computed.

\section{D.Evaluation of the sensitivity of the output load voltage}

Since the response of the circuit corresponding to each topology depends on the matrix exponential and it is difficult to evaluate the sensitivity of the output load voltage with respect to both the input voltage and the load resistance based on the matrix exponential, the Cayley Hamilton Theorem is applied. Suppose that $\mathbf{A}_{i}$ for $i=1,2, \cdots, N$ are diagonalizable. Denote the eigenvalues of $\mathbf{A}_{i}$ as $\lambda_{i, k}$ for $k=1,2, \cdots, n$ and for $i=1,2, \cdots, N$. Define

$$
\left[\begin{array}{c}
\alpha_{i, 1}(t) \\
\vdots \\
\alpha_{i, n}(t)
\end{array}\right] \equiv\left[\begin{array}{ccc}
1 & \cdots & \lambda_{i, 1}^{n-1} \\
\vdots & \ddots & \vdots \\
1 & \cdots & \lambda_{i, n}^{n-1}
\end{array}\right]^{-1}\left[\begin{array}{c}
e^{\lambda_{i, t}} \\
\vdots \\
e^{\lambda_{i, n}}
\end{array}\right]
$$

and

$$
\left[\begin{array}{ccc}
\kappa_{i, 1,1} & \cdots & \kappa_{i, 1, n} \\
\vdots & \ddots & \vdots \\
\kappa_{i, n, 1} & \cdots & \kappa_{i, n, n}
\end{array}\right] \equiv\left[\begin{array}{ccc}
1 & \cdots & \lambda_{i, 1}^{n-1} \\
\vdots & \ddots & \vdots \\
1 & \cdots & \lambda_{i, n}^{n-1}
\end{array}\right]^{-1}
$$

for $t \in\left[t_{i-1}, t_{i}\right)$ and for $i=1,2, \cdots, N$. Then by the Cayley Hamilton Theorem,

$$
e^{\mathbf{A}_{i} t}=\sum_{j=1}^{n} \alpha_{i, j}(t) \mathbf{A}_{i}^{j-1}=\sum_{j=1}^{n} \sum_{k=1}^{n} \kappa_{i, j, k} e^{\lambda_{i, k} t} \mathbf{A}_{i}^{j-1}
$$

for $t \in\left[t_{i-1}, t_{i}\right)$ and for $i=1,2, \cdots, N$. Hence,

$$
\begin{aligned}
& \mathbf{x}(t)=\sum_{j=1}^{n} \sum_{k=1}^{n} \kappa_{i, j, k} e^{\lambda_{i, k}\left(t-t_{i-1}\right)} \mathbf{A}_{i}^{j-1} \mathbf{x}\left(t_{i-1}\right)+ \\
& \int_{t_{i-1}}^{t} \sum_{j=1}^{n} \sum_{k=1}^{n} \kappa_{i, j, k} e^{\lambda_{i, k}(t-\tau)} \mathbf{A}_{i}^{j-1} \mathbf{B}_{i} \mathbf{u}(\tau) d \tau
\end{aligned}
$$

and

$$
\begin{aligned}
& y(t)=\mathbf{C}_{i} \sum_{j=1}^{n} \sum_{k=1}^{n} \kappa_{i, j, k} e^{\lambda_{i, k}\left(t-t_{i-1}\right)} \mathbf{A}_{i}^{j-1} \mathbf{x}\left(t_{i-1}\right)+ \\
& \mathbf{C}_{i} \int_{t_{i-1}}^{t} \sum_{j=1}^{n} \sum_{k=1}^{n} \kappa_{i, j, k} e^{\lambda_{i, k}(t-\tau)} \mathbf{A}_{i}^{j-1} \mathbf{B}_{i} \mathbf{u}(\tau) d \tau+\mathbf{D}_{i} \mathbf{u}(t)
\end{aligned}
$$

for $t \in\left[t_{i-1}, t_{i}\right)$ and for $i=1,2, \cdots, N$. Consequently,

$$
\begin{aligned}
& \mathbf{x}\left(t_{i}-0\right)=\sum_{j=1}^{n} \sum_{k=1}^{n} \kappa_{i, j, k} e^{\lambda_{i, k}\left(t_{i}-t_{i-1}\right)} \mathbf{A}_{i}^{j-1} \mathbf{x}\left(t_{i-1}\right)+ \\
& \int_{t_{i-1}}^{t_{i}-0} \sum_{j=1}^{n} \sum_{k=1}^{n} \kappa_{i, j, k} e^{\lambda_{i, k}\left(t_{i}-\tau\right)} \mathbf{A}_{i}^{j-1} \mathbf{B}_{i} \mathbf{u}(\tau) d \tau
\end{aligned}
$$

and

$$
\begin{aligned}
& y\left(t_{i}-0\right)=\mathbf{C}_{i} \sum_{j=1}^{n} \sum_{k=1}^{n} \kappa_{i, j, k} e^{\lambda_{i, k}\left(t_{i}-t_{i-1}\right)} \mathbf{A}_{i}^{j-1} \mathbf{x}\left(t_{i-1}\right)+ \\
& \mathbf{C}_{i} \int_{t_{i-1}}^{t_{i}-0} \sum_{j=1}^{n} \sum_{k=1}^{n} \kappa_{i, j, k} e^{\lambda_{i, k}\left(t_{i}-\tau\right)} \mathbf{A}_{i}^{j-1} \mathbf{B}_{i} \mathbf{u}(\tau) d \tau+\mathbf{D}_{i} \mathbf{u}\left(t_{i}-0\right)
\end{aligned}
$$

for $i=1,2, \cdots, N-1$. Denote

$$
\widetilde{\mathbf{D}}_{i}(t) \equiv\left[\begin{array}{lll}
\widetilde{d}_{i, 1}(t) & \cdots & \widetilde{d}_{i, p}(t)
\end{array}\right]^{T} \equiv \mathbf{C}_{i} \int_{t_{i-1}}^{t} \sum_{j=1}^{n} \sum_{k=1}^{n} \kappa_{i, j, k} e^{\lambda_{i, k}(t-\tau)} \mathbf{A}_{i}^{j-1} \mathbf{B}_{i} d \tau
$$

for $t \in\left[t_{i-1}, t_{i}\right)$ and for $i=1,2, \cdots, N$, and denote

$$
\mathbf{D}_{i} \equiv\left[\begin{array}{lll}
d_{i, 1} & \cdots & d_{i, p}
\end{array}\right]^{T} \text { for } i=1,2, \cdots, N
$$

Then

$\frac{\partial y(t)}{\partial u_{k}(t)} \approx \widetilde{d}_{i, k}(t)+d_{i, k} \quad \forall t \in\left[t_{i-1}, t_{i}\right)$, for $i=1,2, \cdots, N$ and for $k=1,2, \cdots, p$.

Denote $\mathbf{A}_{i}^{j-1} \equiv\left[\begin{array}{ccc}a_{1,1}^{i, j-1} & \cdots & a_{1, n}^{i, j-1} \\ \vdots & \ddots & \vdots \\ a_{n, 1}^{i, j-1} & \cdots & a_{n, n}^{i, j-1}\end{array}\right]$ for $i=1,2, \cdots, N$ and for $j=1,2, \cdots, n$. Denote $\mathbf{B}_{i} \equiv\left[\begin{array}{ccc}b_{i, 1,1} & \cdots & b_{i, 1, p} \\ \vdots & \ddots & \vdots \\ b_{i, n, 1} & \cdots & b_{i, n, p}\end{array}\right], \mathbf{C}_{i} \equiv\left[\begin{array}{lll}c_{i, 1} & \cdots & c_{i, n}\end{array}\right]$ and $\mathbf{x}_{i}\left(t_{i-1}\right) \equiv\left[\begin{array}{lll}x_{i, 1}\left(t_{i-1}\right) & \cdots & x_{i, n}\left(t_{i-1}\right)\end{array}\right]^{T}$ for $i=1,2, \cdots, N$. Then:

$$
\begin{gathered}
\mathbf{C}_{i} \sum_{j=1}^{n} \sum_{k=1}^{n} \kappa_{i, j, k} e^{\lambda_{i, k}\left(t-t_{i-1}\right)} \mathbf{A}_{i}^{j-1} \mathbf{x}\left(t_{i-1}\right)=\sum_{m=1}^{n} \sum_{l=1}^{n} \sum_{j=1}^{n} \sum_{k=1}^{n} c_{i, l} \kappa_{i, j, k} e^{\lambda_{i, k}\left(t-t_{i-1}\right)} a_{l, m}^{i, j-1} x_{i, m}\left(t_{i-1}\right), \\
\mathbf{C}_{i} \int_{t_{i-1}}^{t} \sum_{j=1}^{n} \sum_{k=1}^{n} \kappa_{i, j, k} e^{\lambda_{i, k}(t-\tau)} \mathbf{A}_{i}^{j-1} \mathbf{B}_{i} \mathbf{u}(\tau) d \tau= \\
\sum_{q=1}^{p} \sum_{m=1}^{n} \sum_{l=1}^{n} \sum_{j=1}^{n} \sum_{k=1}^{n} \int_{t_{i-1}}^{t} c_{i, l} \kappa_{i, j, k} e^{\lambda_{i, k}(t-\tau)} a_{l, m}^{i, j-1} b_{i, m, q} u_{q}(\tau) d \tau
\end{gathered}
$$

and $\mathbf{D}_{i} \mathbf{u}(t)=\sum_{q=1}^{p} d_{q} u_{q}(t) \quad \forall t \in\left[t_{i-1}, t_{i}\right)$ and for $i=1,2, \cdots, N$.

Hence,

$$
\begin{aligned}
& \frac{\partial}{\partial R_{L}} \mathbf{C}_{i} \sum_{j=1}^{n} \sum_{k=1}^{n} \kappa_{i, j, k} e^{\lambda_{i, k}\left(t-t_{i-1}\right)} \mathbf{A}_{i}^{j-1} \mathbf{x}\left(t_{i-1}\right)= \\
& \sum_{m=1}^{n} \sum_{l=1}^{n} \sum_{j=1}^{n} \sum_{k=1}^{n} c_{i, l} \kappa_{i, j, k} e^{\lambda_{i, k}\left(t-t_{i-1}\right)}\left(\frac{\partial a_{l, m}^{i, j-1}}{\partial R_{L}}\right) x_{i, m}\left(t_{i-1}\right)+ \\
& \sum_{m=1}^{n} \sum_{l=1}^{n} \sum_{j=1}^{n} \sum_{k=1}^{n} c_{i, l} \kappa_{i, j, k}\left(t-t_{i-1}\right)\left(\frac{\partial \lambda_{i, k}}{\partial R_{L}}\right) e^{\lambda_{i, k}\left(t-t_{i-1}\right)} a_{l, m}^{i, j-1} x_{i, m}\left(t_{i-1}\right)+
\end{aligned}
$$




$$
\begin{aligned}
& \sum_{m=1}^{n} \sum_{l=1}^{n} \sum_{j=1}^{n} \sum_{k=1}^{n} c_{i, l}\left(\frac{\partial \kappa_{i, j, k}}{\partial R_{L}}\right) e^{\lambda_{i, k}\left(t-t_{i-1}\right)} a_{l, m}^{i, j-1} x_{i, m}\left(t_{i-1}\right)+ \\
& \sum_{m=1}^{n} \sum_{l=1}^{n} \sum_{j=1}^{n} \sum_{k=1}^{n}\left(\frac{\partial c_{i, l}}{\partial R_{L}}\right) \kappa_{i, j, k} e^{\lambda_{i, k}\left(t-t_{i-1}\right)} a_{l, m}^{i, j-1} x_{i, m}\left(t_{i-1}\right)
\end{aligned}
$$

$\frac{\partial}{\partial R_{L}} \mathbf{C}_{i} \int_{t_{i-1}}^{t} \sum_{j=1}^{n} \sum_{k=1}^{n} \kappa_{i, j, k} e^{\lambda_{i, k}(t-\tau)} \mathbf{A}_{i}^{j-1} \mathbf{B}_{i} \mathbf{u}(\tau) d \tau=$

$\sum_{q=1}^{p} \sum_{m=1}^{n} \sum_{l=1}^{n} \sum_{j=1}^{n} \sum_{k=1}^{n} \int_{t_{i-1}}^{t}\left(\frac{\partial c_{i, l}}{\partial R_{L}}\right) \kappa_{i, j, k} e^{\lambda_{i, k}(t-\tau)} a_{l, m}^{i, j-1} b_{i, m, q} u_{q}(\tau) d \tau+$

$\sum_{q=1}^{p} \sum_{m=1}^{n} \sum_{l=1}^{n} \sum_{j=1}^{n} \sum_{k=1}^{n} \int_{t_{i-1}}^{t} c_{i, l}\left(\frac{\partial \kappa_{i, j, k}}{\partial R_{L}}\right) e^{\lambda_{i, k}(t-\tau)} a_{l, m}^{i, j-1} b_{i, m, q} u_{q}(\tau) d \tau+$

$\sum_{q=1}^{p} \sum_{m=1}^{n} \sum_{l=1}^{n} \sum_{j=1}^{n} \sum_{k=1}^{n} \int_{t_{i-1}}^{t} c_{i, l} \kappa_{i, j, k}(t-\tau)\left(\frac{\partial \lambda_{i, k}}{\partial R_{L}}\right) e^{\lambda_{i, k}(t-\tau)} a_{l, m}^{i, j-1} b_{i, m, q} u_{q}(\tau) d \tau+$

$\sum_{q=1}^{p} \sum_{m=1}^{n} \sum_{l=1}^{n} \sum_{j=1}^{n} \sum_{k=1}^{n} \int_{t_{i-1}}^{t} c_{i, l} \kappa_{i, j, k} e^{\lambda_{i, k}(t-\tau)}\left(\frac{\partial a_{l, m}^{i, j-1}}{\partial R_{L}}\right) b_{i, m, q} u_{q}(\tau) d \tau+$

$\sum_{q=1}^{p} \sum_{m=1}^{n} \sum_{l=1}^{n} \sum_{j=1}^{n} \sum_{k=1}^{n} \int_{t_{i-1}}^{t} c_{i, l} \kappa_{i, j, k} e^{\lambda_{i, k}(t-\tau)} a_{l, m}^{i, j-1}\left(\frac{\partial b_{i, m, q}}{\partial R_{L}}\right) u_{q}(\tau) d \tau$

and $\frac{\partial}{\partial R_{L}} \mathbf{D}_{i} \mathbf{u}(t)=\sum_{q=1}^{p}\left(\frac{\partial d_{q}}{\partial R_{L}}\right) u_{q}(t) \quad \forall t \in\left[t_{i-1}, t_{i}\right)$ and for

$i=1,2, \cdots, N$. Consequently, we have:

$$
\begin{aligned}
& \frac{\partial y(t)}{\partial R_{L}}=\sum_{m=1}^{n} \sum_{l=1}^{n} \sum_{j=1}^{n} \sum_{k=1}^{n} c_{i, l} \kappa_{i, j, k} e^{\lambda_{i, k}\left(t-t_{i-1}\right)}\left(\frac{\partial a_{l, m}^{i, j-1}}{\partial R_{L}}\right) x_{i, m}\left(t_{i-1}\right)+ \\
& \sum_{m=1}^{n} \sum_{l=1}^{n} \sum_{j=1}^{n} \sum_{k=1}^{n} c_{i, l} \kappa_{i, j, k}\left(t-t_{i-1}\right)\left(\frac{\partial \lambda_{i, k}}{\partial R_{L}}\right) e^{\lambda_{i, k}\left(t-t_{i-1}\right)} a_{l, m}^{i, j-1} x_{i, m}\left(t_{i-1}\right)+ \\
& \sum_{m=1}^{n} \sum_{l=1}^{n} \sum_{j=1}^{n} \sum_{k=1}^{n} c_{i, l}\left(\frac{\partial \kappa_{i, j, k}}{\partial R_{L}}\right) e^{\lambda_{i, k}\left(t-t_{i-1}\right)} a_{l, m}^{i, j-1} x_{i, m}\left(t_{i-1}\right)+ \\
& \sum_{m=1}^{n} \sum_{l=1}^{n} \sum_{j=1}^{n} \sum_{k=1}^{n}\left(\frac{\partial c_{i, l}}{\partial R_{L}}\right) \kappa_{i, j, k} e^{\lambda_{i, k}\left(t-t_{i-1}\right)} a_{l, m}^{i, j-1} x_{i, m}\left(t_{i-1}\right)+ \\
& \sum_{q=1}^{p} \sum_{m=1}^{n} \sum_{l=1}^{n} \sum_{j=1}^{n} \sum_{k=1}^{n} \int_{t_{i-1}}^{t}\left(\frac{\partial c_{i, l}}{\partial R_{L}}\right) \kappa_{i, j, k} e^{\lambda_{i, k}(t-\tau)} a_{l, m}^{i, j-1} b_{i, m, q} u_{q}(\tau) d \tau+ \\
& \sum_{q=1}^{p} \sum_{m=1}^{n} \sum_{l=1}^{n} \sum_{j=1}^{n} \sum_{k=1}^{n} \int_{t_{i-1}}^{t} c_{i, l}\left(\frac{\partial \kappa_{i, j, k}}{\partial R_{L}}\right) e^{\lambda_{i, k}(t-\tau)} a_{l, m}^{i, j-1} b_{i, m, q} u_{q}(\tau) d \tau+ \\
& \sum_{q=1}^{p} \sum_{m=1}^{n} \sum_{l=1}^{n} \sum_{j=1}^{n} \sum_{k=1}^{n} \int_{t_{i-1}}^{t} c_{i, l} \kappa_{i, j, k}(t-\tau)\left(\frac{\partial \lambda_{i, k}}{\partial R_{L}}\right) e^{\lambda_{i, k}(t-\tau)} a_{l, m}^{i, j-1} b_{i, m, q} u_{q}(\tau) d \tau+ \\
& \sum_{q=1}^{p} \sum_{m=1}^{n} \sum_{l=1}^{n} \sum_{j=1}^{n} \sum_{k=1}^{n} \int_{t_{i-1}}^{t} c_{i, l} \kappa_{i, j, k} e^{\lambda_{i, k}(t-\tau)}\left(\frac{\partial a_{l, m}^{i, j-1}}{\partial R_{L}}\right) b_{i, m, q} u_{q}(\tau) d \tau+ \\
& \sum_{q=1}^{p} \sum_{m=1}^{n} \sum_{l=1}^{n} \sum_{j=1}^{n} \sum_{k=1}^{n} \int_{t_{i-1}}^{t} c_{i, l} \kappa_{i, j, k} e^{\lambda_{i, k}(t-\tau)} a_{l, m}^{i, j-1}\left(\frac{\partial b_{i, m, q}}{\partial R_{L}}\right) u_{q}(\tau) d \tau+ \\
& \sum_{q=1}^{p}\left(\frac{\partial d_{q}}{\partial R_{L}}\right) u_{q}(t) \\
& \forall t \in\left[t_{i-1}, t_{i}\right) \text { and for } i=1,2, \cdots, N \text {. }
\end{aligned}
$$

By substituting (10) into Problem (R), the problem can be solved efficiently via many CAD tools, such as MISER3 [30].

\section{ILLUSTRATIVE EXAMPLE}

Since few works have been done on the determination of the switching instants based on minimizing the output ripple magnitude, the output leakage voltage and the sensitivity of the output load voltage with respect to both the input voltage and the load resistance, it is difficult to have a fair comparison. This is because it is unfair if we compare this optimal PWM control method to those control methods with different control strategies (such as those methods which do not determine the optimal switching instants, but determine the optimal control input signals), non-optimal control methods (such as those methods in which the switching instants are not optimal), or optimal control methods with different cost functions and constraints (such as those methods in which the switching instants are optimized based on other criteria, but not on the minimization of the output ripple magnitude, the output leakage voltage and the sensitivity of the output load voltage with respect to both the input voltage and the load resistance). In order to illustrate the effectiveness of this optimal PWM control method, we consider a switched-capacitor DC/DC power converter discussed in [31] as an illustrative example. This switched-capacitor DC/DC power converter [31] is chosen because of its simplicity and having practical applications in industries.

Referring to the example in [31] which switches between two topologies, the circuit corresponding to each topology is characterized by an affine linear time invariant state space model as follows:

$$
\begin{aligned}
& \mathbf{A}_{1}=\frac{1}{\alpha_{1}}\left[\begin{array}{ccc}
-\frac{\alpha_{1}}{C_{1}\left(R_{C 1}+2 R_{\text {on }}\right)} & 0 & 0 \\
0 & -\frac{R_{L}+R_{C 0}}{C_{2}} & \frac{R_{L}}{C_{2}} \\
0 & \frac{R_{L}}{C_{0}} & -\frac{R_{L}+R_{C 2}+2 R_{\text {on }}}{C_{0}}
\end{array}\right] \\
& \mathbf{A}_{2}=\frac{1}{\alpha_{2}}\left[\begin{array}{ccc}
-\frac{R_{L}+R_{C 0}}{C_{1}} & 0 & \frac{R_{L}}{C_{1}} \\
0 & -\frac{\alpha_{2}}{C_{2}\left(R_{C 2}+2 R_{o n}\right)} & 0 \\
\frac{R_{L}}{C_{0}} & 0 & -\frac{R_{L}+R_{C 1}+2 R_{o n}}{C_{0}}
\end{array}\right] \text {, }
\end{aligned}
$$

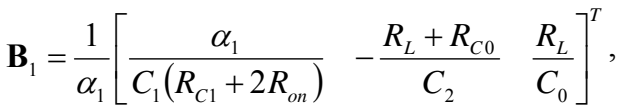

$$
\begin{aligned}
& \mathbf{B}_{2}=\frac{1}{\alpha_{2}}\left[\begin{array}{lll}
-\frac{R_{L}+R_{C 0}}{C_{1}} & \frac{\alpha_{2}}{C_{2}\left(R_{C 2}+2 R_{\text {on }}\right)} & \frac{R_{L}}{C_{0}}
\end{array}\right]^{T}, \\
& \mathbf{C}_{1}=\frac{1}{\alpha_{1}}\left[\begin{array}{lll}
0 & R_{L} R_{C 0} & R_{L}\left(R_{C 2}+2 R_{\text {on }}\right)
\end{array}\right] \text {, } \\
& \mathbf{C}_{2}=\frac{1}{\alpha_{2}}\left[R_{L} R_{C 0} \quad 0 \quad R_{L}\left(R_{C 1}+2 R_{\text {on }}\right)\right] \text {, } \\
& D_{1}=\frac{R_{C 0} R_{L}}{\alpha_{1}},
\end{aligned}
$$

and

$$
D_{2}=\frac{R_{C 0} R_{L}}{\alpha_{2}}
$$

where 


$$
\alpha_{1}=R_{C 0} R_{L}+\left(R_{C 2}+2 R_{o n}\right)\left(R_{L}+R_{C 0}\right)
$$

and

$$
\alpha_{2}=R_{C 0} R_{L}+\left(R_{C 1}+2 R_{\text {on }}\right)\left(R_{L}+R_{C 0}\right) .
$$

In this example, $N=2$. Figure 1 shows the circuit schematics corresponding to each topology. The values of the resistors and capacitors, as well as the input voltage, are shown in the circuit schematic, where $R_{\text {on }}=0.01 \Omega$ for all the diodes and transistors. We operate the circuit with switching frequency equal to $550 \mathrm{kHz}$, that is $T=t_{N}=1.8182 \times 10^{-6} \mathrm{~s}$. We select this switching frequency because there will be a strong electronic magnetic interference and large ripple magnitudes if we operate the circuit at higher or lower switching frequencies. We assume that the circuit is initially at rest, that is $\mathbf{x}(0)=\mathbf{0}$, as the usual case in the real situation. All the weights for combining the multiple-objectives are chosen to be 1 to avoid having bias on a particular objective. Since the output leakage voltage is smaller than the output ripple magnitude, we neglect the effect of the output leakage voltage in the cost function, that is, $y\left(t_{1}\right)=y\left(t_{1}-0\right)$. By applying this optimal PWM control method, we found that $t_{1}=1.5209 \times 10^{-6} \mathrm{~s}$ and the output ripple magnitude is $3.6242 \times 10^{-4} \mathrm{~V}$. The corresponding duty cycle is 0.8365. Although $C_{1}=C_{2}$ and $R_{C 1}=R_{C 2}$, it is worth noting that the optimal duty cycle does not operate at 0.5 . This is because the charging time constant is different from the discharging time constant due to the non-zero load resistance as well as parasitic switch resistance and capacitance. Both the transient and steady state responses of all capacitors, as well as the output load voltages are shown in Figure 2. It can be seen from the figure that the steady state voltages across $C_{0}, C_{1}, C_{2}$ and $R_{L}$ are approximately, $3.6 \mathrm{~V}, 1.8 \mathrm{~V}, 1.8 \mathrm{~V}$ and $3.6 \mathrm{~V}$, respectively. Hence, the circuit behaves normally as a voltage doubler.

\section{CONCLUSIONS}

In this paper, we apply the model transformation and the enhancing control methods to determine the optimal switching instants for PWM control of switched-capacitor DC/DC power converters. The advantages of applying this optimal PWM control method are to avoid the process of a linearization and guarantee the optimality. Compared to the existing PWM control methods, these methods are only a local approximation of the problem and do not solve the problem completely.

\section{REFERENCES}

[1] TianRui Ying, Wing-Hung Ki and Mansun Chan, "Area-efficient CMOS charge pumps for LCD drivers," IEEE Journal of Solid-State Circuits, vol. 38, no. 10, pp. 1721-1725, 2003.

[2] Jian Liu, Zhiming Chen and Zhong Du, "A new design of power supplies for pocket computer systems," IEEE Transactions on Industrial Electronics, vol. 45, no. 2, pp. 228-235, 1998.

[3] Octavian Dranga, Balázs Buti and István Nagy, "Stability analysis of a feedback-controlled resonant DC-DC converter," IEEE Transactions on Industrial Electronics, vol. 50, no. 1, pp. 141-152, 2003.

[4] Sudip K. Mazumder, Ali H. Nayfeh and Dushan Boroyevich, "Theoretical and experimental investigation of the fast- and slow-scale instabilities of a
DC-DC converter," IEEE Transactions on Power Electronics, vol. 16, no. 2, pp. 201-216, 2001.

[5] Yefim Berkovich and Adrian Ioinovici, "Large-signal stability-oriented design of boost regulators based on a Lyapunov criterion with nonlinear integral," IEEE Transactions on Circuits and Systems-1: Fundamental Theory and Applications, vol. 49, no. 11, pp. 1610-1619, 2002.

[6] Soumitro Banerjee and Krishnendu Chakrabarty, "Nonlinear modeling and bifurcations in the boost converter," IEEE Transactions on Power Electronics, vol. 13, no. 2, pp. 252-260, 1998.

[7] David C. Hamill, Jonathan H. B. Deane and David J. Jefferies, "Modeling of chaotic DC-DC converters by iterated nonlinear mappings," IEEE Transactions on Power Electronics, vol. 7, no. 1, pp. 25-36, 1992.

[8] Guohui Yuan, Soumitro Banerjee, Edward Ott and James A. Yorke, "Border-collision bifurcations in the buck converter," IEEE Transactions on Circuits and Systems-I: Fundamental Theory and Applications, vol. 45, no. 7, pp. 707-716, 1998.

[9] Mario di Bernardo and Francesco Vasca, "Discrete-time maps for the analysis of bifurcations and chaos in DC/DC converters," IEEE Transactions on Circuits and Systems - Part I: Fundamental Theory and Applications, vol. 47, no. 2, pp. 130-143, 2000.

[10] K. W. E. Cheng, M. Liu and J. Wu, "Experimental study of bifurcation and chaos in the buck-boost converter," IEE Proceedings. B, Electric Power Applications, vol. 150, no. 1, pp. 45-61, 2003.

[11] William C. Y. Chan and Chi K. Tse, "Study of bifurcations in current-programmed DC/DC boost converters: from quasi-periodicity to period-doubling," IEEE Transactions on Circuits and Systems- $\mathrm{I}$ : Fundamental Theory and Applications, vol. 44, no. 12, pp. 1129-1142, 1997.

[12] C. K. Tse, S. C. Fung and M. W. Kwan, "Experimental confirmation of chaos in a current-programmed Ćuk converter," IEEE Transactions on Circuits and Systems - I: Fundamental Theory and Applications, vol. 43, no. 7, pp. 605-608, 1996.

[13] K. W. E. Cheng, M. Liu and J. Wu, "Chaos study and parameter-space analysis of the DC-DC buck-boost converter," IEE Proceedings. B, Electric Power Applications, vol. 150, no. 2, pp. 126-138, 2003.

[14] Carles Batlle, Imma Massana and Alicia Miralles, "Lyapunov exponents for bilinear systems. Application to the buck converter," International Journal of Bifurcation and Chaos, vol. 13, no. 3, pp. 713-722, 2003.

[15] David C. Hamill and David J. Jeffries, "Subharmonics and chaos in a controlled switched-mode power converter," IEEE Transactions on Circuits and Systems, vol. 35, no. 8, pp. 1059-1061, 1988.

[16] Alireza Khayatian and David G. Taylor, "Multirate modeling and control design for switched-mode power converters," IEEE Transactions on Automatic Control, vol. 39, no. 9, pp. 1848-1852, 1994.

[17] Frank H. F. Leung, Peter K. S. Tam and C. K. Li, "An improved LQR-based controller for switching DC-DC converters," IEEE Transactions on Industrial Electronics, vol. 40, no. 5, pp. 521-528, 1993.

[18] Frank H. F. Leung, Peter K. S. Tam and C. K. Li, "The control of switching DC-DC converters-A general LQR problem," IEEE Transactions on Industrial Electronics, vol. 38, no. 1, pp. 65-71, 1991.

[19] Francesco Garofalo, Pompeo Marino, Stefano Scala and Francesco Vasca, "Control of DC-DC converters with linear optimal feedback and nonlinear feedforward," IEEE Transactions on Power Electronics, vol. 9, no. 6 , pp. 607-615, 1994.

[20] J. Sun, H. Takano and M. Nakaoka, "Series and parallel transformer resonant DC-DC converter using optimal digital servo and repetitive learning control schemes," IEE Proceedings. B, Electric Power Applications, vol. 146, no. 5, pp. 530-538, 1999.

[21] Henry Chung and Adrian Ioinovici, "Design of feedback gain vector of two-state basic PWM multifeedback regulators for large-signal stability," IEEE Transactions on Circuits and Systems- I: Fundamental Theory and Applications, vol. 44, no. 8, pp. 676-683, 1997.

[22] Yufei Zhou, Chi K. Tse, Shui-Sheng Qiu and Francis C. M. Lau, "Applying resonant parametric perturbation to control chaos in the buck DC/DC converter with phase shift and frequency mismatch considerations," International Journal of Bifurcation and Chaos, vol. 13, no. 11 , pp. 3459-3471, 2003.

[23] L. Benadero, A. El Aroudi, G. Olivar, E. Toribio and E. Gómez, "Two-dimensional bifurcation diagrams. Background pattern of fundamental DC-DC converters with PWM control," International Journal of Bifurcation and Chaos, vol. 13, no. 2, pp. 427-451, 2003. 
[24] Gabriel Garcerá, Emilio Figueres and Antonio Mocholí, "Novel three-controller average current mode control of DC-DC PWM converters with improved robustness and dynamic response," IEEE Transactions on Power Electronics, vol. 15, no. 3, pp. 516-528, 2000.

[25] Luigi Calderone, Licia Pinola and Vincenzo Varoli, "Optimal feed-forward compensation for PWM DC/DC converters with "linear" and "quadratic" conversion ratio," IEEE Transactions on Power Electronics, vol. 7, no. 2, pp. 349-355, 1992.

[26] K. L. Teo, C. J. Goh and K. H. Wong, A unified computational approach to optimal control problems, vol. 55, Pitman Monographs and Surveys in Pure and Applied Mathematics (Longman Scientific and Technical, England, 1991).

[27] K. L. Teo, L. S. Jennings, H. W. J. Lee and V. Rehbock, "The control parameterization enhancing transform for constrained optimal control problems," Journal of Australian Mathematical Society, Series B, vol. 40, pp. 314-335, 1999.

[28] X. Q. Yang, A. I. Mees, M. E. Fisher and L. S. Jennings, Progress in Optimization II Contributions from Australasia.

[29] Y. Liu, K. L. Teo, L. S. Jennings and S. Wang, "On a class of optimal control problems with state jumps," Journal of Optimization Theory and Applications, vol. 98, no. 1, pp. 65-82, 1998.

[30] L. S. Jennings, M. E. Fisher, K. L. Teo and C. J. Goh, "MISER3: Solving optimal control problems - an update," Advances in Engineering Software, vol. 13, pp. 190-196, 1991.

[31] Henry Chung and Y. K. Mok, "Development of a switched-capacitor DC/DC boost converter with continuous input current waveform," IEEE Transactions on Circuits and Systems-1: Fundamental Theory and Applications, vol. 46, no. 6, pp. 756-759, 1999.

[32] Guangyong Zhu and Adrian Ioinovici, "DC-to-DC converter with no magnetic elements and enhanced regulation," IEEE Transactions on Aerospace and Electronic Systems, vol. 33, no. 2, pp. 499-506, 1997.

[33] Arun Rao, William McIntyre, Un-Ku Moon and Gábor C. Temes, "Noise-shaping techniques applied to switched-capacitor voltage regulators," IEEE Journal of Solid-State Circuits, vol. 40, no. 2, pp. 422-429, 2005.

[34] Hoi Lee and Philip K. T. Mok, "An SC voltage doubler with pseudo-continuous output regulation using a three-stage switchable Opamp," IEEE Journal of Solid-State Circuits, vol. 42, no. 6, pp. 1216-1229, 2007.

[35] Jifeng Han, Annette von Jouanne and Gábor C. Temes, "A new approach to reducing output ripple in switched-capacitor-based step-down DC-DC converters," IEEE Transactions on Power Electronics, vol. 21, no. 6, pp. 1548-1555, 2006.

[36] B. Robert Gregoire, "A compact switched-capacitor regulated charge pump power supply,” IEEE Journal of Solid-State Circuits, vol. 41, no. 8, pp. 1944-1953, 2006.
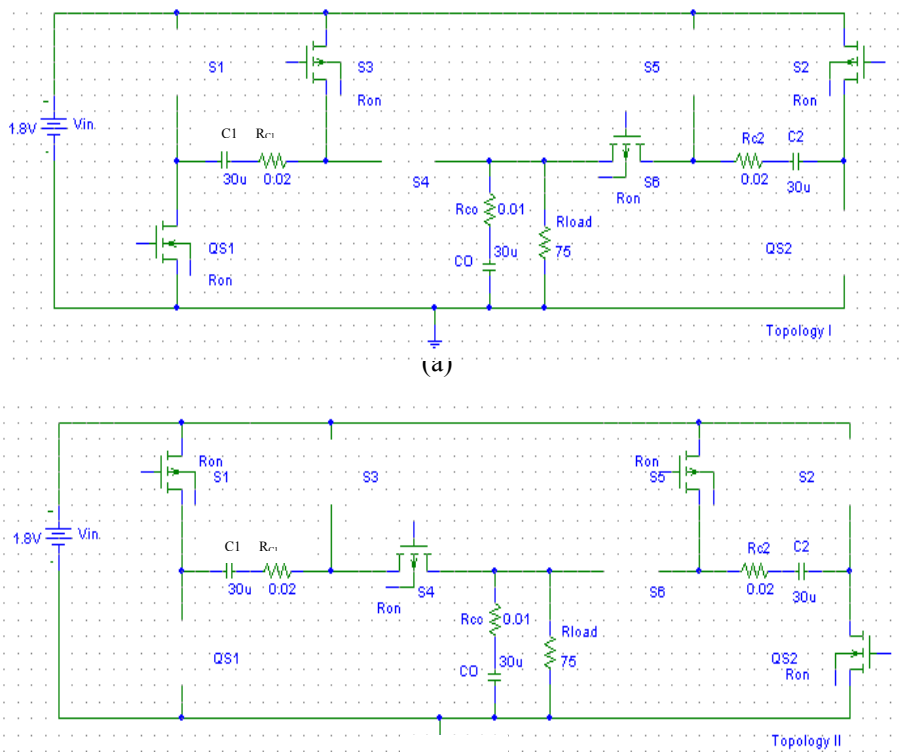

(b)
Figure 1. Circuit schematics of each topology of the switched-capacitor DC/DC power converter [31]. (a) Topology 1. (b) Topology 2.
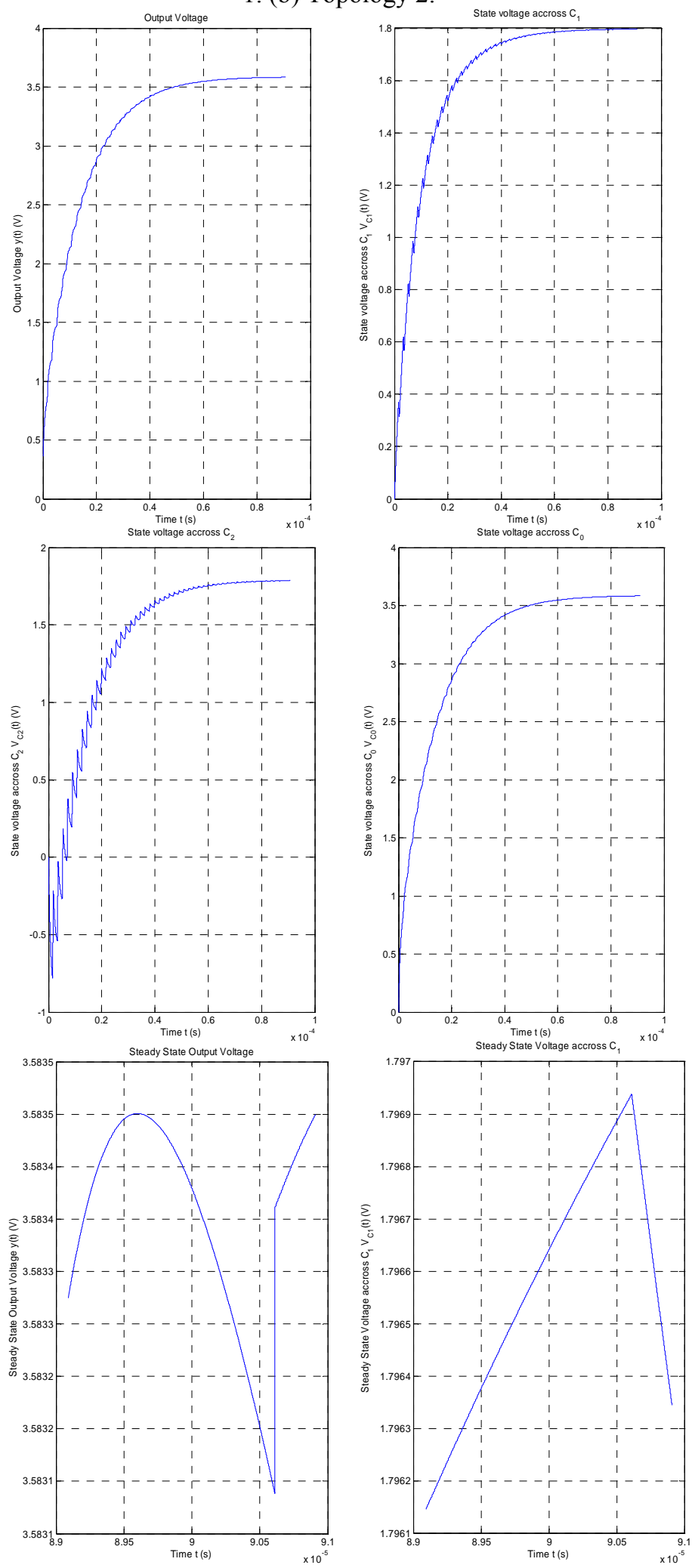

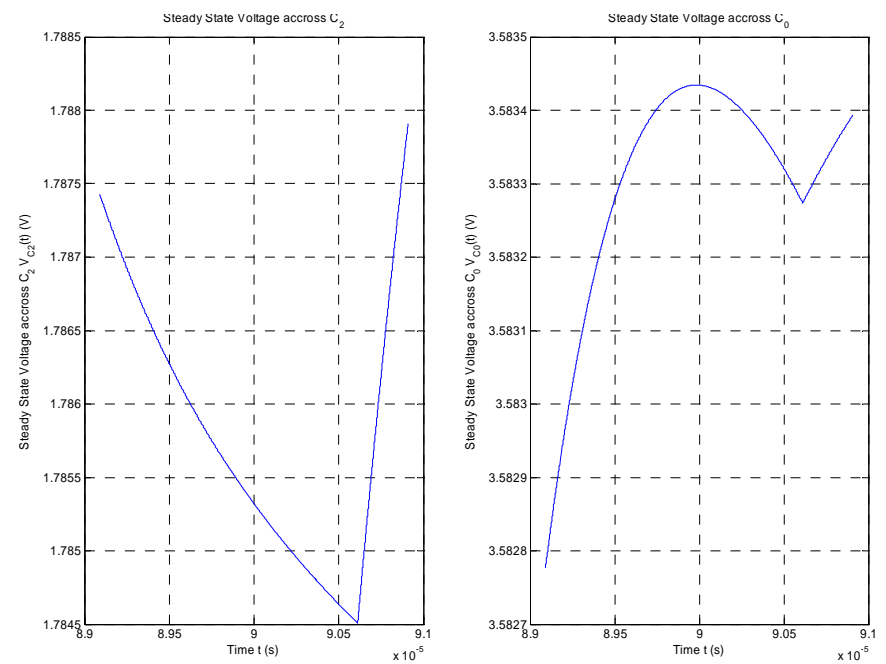

Figure 2. Transient and steady state voltages across capacitors and the load resistor of the switched-capacitor DC/DC power converter [31]. 
